\title{
Skin Response Specimen Type
}

National Cancer Institute

\section{Source}

National Cancer Institute. Skin Response Specimen Type. NCI Thesaurus. Code C117687.

The type of a material sample taken from a biological entity for skin response testing. 